\title{
An Investigation on using Lagrange, Newton and Least Square Methods for Generating Nonlinear Interpolation Function for the Measuring Instruments
}

\author{
Gouda M. Mahmoud ${ }^{*}$ and Shaker A. Gelany ${ }^{1}$

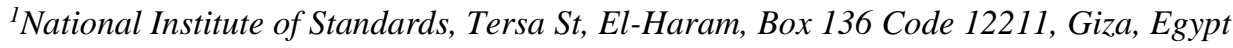

\begin{abstract}
This research is considered the milestone for metrologists to choose the appropriate method for determination of the nonlinear interpolation function for the measuring instruments. Three methods of generating the interpolation polynomial equations were investigated; Newton, Lagrange, and Least Square method. The response of the measuring instruments under investigation was calculated and compared with the experimental results. Least Square method was found that it is the most accurate and most realistic approach to determine the interpolation polynomial function for the measuring instruments. It is recommended to use Least Square method rather than other methods to interpolating the polynomial equation. This recommendation is very important for metrologist as well as for measuring instruments applicant. This article is millstone to determine the response of the measuring instrument at non calibrated points in the calibrated range.
\end{abstract}

Keywords: Lagrange interpolation; Newton interpolation; Least Square interpolation; uncertainty; curve fitting

\section{INTRODUCTION}

Numerical analysis is the area of mathematics and computer science that creates, analyzes, and implements a numerical method for solving the problems of continuous mathematic numerically (Srivastava \& Purushottam 2012). In the field of numerical analysis, interpolation is a type of estimation, a method for constructing new data points within the range of a discrete set of known values. Interpolation is also defined as an estimation of a value within two known points in a sequence of values. Interpolation plays an important role in modern approximation science. In engineering and science, it is often necessary to interpolate (i.e. estimate) the value of a function for an intermediate value of an independent variable. Interpolation theory has been developed since the seventeenth century when calculus was proposed ( $\mathrm{Li}$ et al., 2016). Polynomial interpolation is a method of estimating values between known data points. When graphical data contains a gap, but data is available on either side of the gap or at a few specific points within the gap, interpolation allows us to estimate the values within the gap. If $x_{0}<\ldots<x_{n}$ and $y_{0}=f\left(x_{0}\right), y_{n}=f\left(x_{n}\right)$ are known, and if $x_{0}<x<x_{n}$, then the estimated values of $f(x)$ is known as an interpolation. If $x<x_{0}$ or $x>x_{n}$, the estimated values of $f(x)$ is known as extrapolation. There are many approaches for interpolation functions determination, such as Least Square Method (LSM), Lagrange Approach (LA) and Newton Approach (NA); those approaches are used in various usage. Those methods are utilized in linear and nonlinear polynomial interpolation. For polynomial interpolation, it is often needed to determine the values of a function $y=f(x)$ at certain point $x$ based on known values of function $f\left(x_{o}\right), \ldots \ldots \ldots f\left(x_{n}\right)$ at a set of $n+1$ node points $a=$ $x_{o} \leq x_{1} \leq \ldots \ldots . \leq x_{n}=b$ in the interval $[\mathrm{a}, \mathrm{b}]$. One way to carry out these operations is to approximate the function $f(x)$ by an $n^{\text {th }}$ degree polynomial (Rainer, 1998):

$$
\begin{aligned}
& f(x) \approx p_{n}(x) \\
& =a_{n} x^{n}+a_{n-1} x^{n-1}+\ldots \ldots a_{2} x^{2}
\end{aligned}
$$




$$
+a_{1} x+a_{o}
$$

Where coefficients $a_{\mathrm{o}}, a_{1}, a_{2} \ldots$, an could be obtained based on $n+1$ given points. Once $p_{n}(x)$ becomes obtainable, any mathematical operations implemented to the function $f(x)$ such as integration, differentiation and root findings, could be executed approximately in accordance with $p_{n}(x) \approx f(x)$. To determine the coefficients of $p_{n}(x)$, it is required to pass through all node points

$\left\{x_{\mathrm{i}}, y_{\mathrm{i}}, \ldots \ldots ..\right\}=f\left(x_{i}\right), \quad i=0, \ldots . . n$, the following $n+1$ linear equations hold (3):

$$
p_{n}\left(x_{i}\right) \sum_{j=0}^{n} a_{j} x_{j}^{i}=f\left(x_{i}\right)=y_{i}, \quad(i=0, \ldots n)
$$

Now the coefficients $a_{0}, \ldots . a_{\mathrm{n}}$ could be obtained by resolving those equations, which can be expressed in matrix form as:

$$
\left[\begin{array}{ccccc}
1 & x_{o} & x_{0}^{2} & \cdots & x_{0}^{n} \\
1 & x_{1} & x_{1}^{2} & \cdots & x_{1}^{n} \\
1 & x_{2} & x_{2}^{2} & \cdots & x_{2}^{n} \\
\vdots & \vdots & \vdots & \cdots & \vdots \\
1 & x_{n} & x_{n}^{2} & \cdots & x_{n}^{n}
\end{array}\right] *\left[\begin{array}{c}
a_{o} \\
a_{1} \\
a_{2} \\
\vdots \\
a_{n}
\end{array}\right]=V a=\left[\begin{array}{c}
y_{o} \\
y_{1} \\
y_{2} \\
\vdots \\
y_{n}
\end{array}\right]=y
$$

Occasionally, we may have to approximate the intermediate values between precise data points, i.e. paired data from the experiment. To deal with this issue the interpolation methods, such as Lagrange interpolation, Newton's interpolation, and Least Square Method interpolation (Han \& Gaik, 2016) was needed.

\section{MATHEMATICAL OVERVIEW}

\section{A. The Newton Polynomial Interpolation}

In Newton interpolation method, more points of intermediate values are to be determined. All nonlinear polynomial functions and their related coefficients remain the same. Due to the supplementary terms, the degrees of ponynomial interpolation functions are increased and the approximations error might be decreased (in case of higher order polynomials interpolation) (Ramesh, 2012). The basic polynomials of the Newton interpolation are determined as follow (Gu et al., 2020)

$$
n_{o}(x)=1, n_{i}(x)=\coprod_{j=0}^{i-1}\left(x-x_{j}\right),(i-1, \ldots, n)
$$

and the Newton interpolating polynomial is constructed:

$$
\begin{aligned}
N_{n}(x)= & \sum c_{i} n_{i}(x) \\
& =c_{o}+\sum_{i=1}^{n} c_{i}\left(\coprod_{j=0}^{i-1}\left(x-x_{j}\right)\right) \\
& =c_{o}+c_{1}\left(x-x_{o}\right)+c_{2}\left(x-x_{o}\right)\left(-x_{1}\right) \\
& +\ldots+c_{n} \coprod_{j=0}^{n-1}\left(x-x_{i}\right)
\end{aligned}
$$

$\left[\begin{array}{cccccc}1 & & & & \\ 1 & \left(x_{1}-x_{o}\right) & & & \\ 1 & \left(x_{2}-x_{o}\right) & \left(x_{2}-x_{o}\right)\left(x_{2}-x_{1}\right) & & \\ 1 & \left(x_{3}-x_{o}\right) & \left(x_{3}-x_{o}\right)\left(x_{3}-x_{1}\right) & \left(x_{3}-x_{o}\right)\left(x_{3}-x_{1}\right)\left(x_{3}-x_{2}\right) & & \\ \vdots & \vdots & \vdots & \vdots & \cdots & \\ 1 & \left(x_{n}-x_{o}\right) & \left(x_{n}-x_{o}\right)\left(x_{n}-x_{1}\right) & \left(x_{n}-x_{o}\right)\left(x_{n}-x_{1}\right)\left(x_{n}-x_{2}\right) & \cdots & \bigsqcup_{i=0}^{n-1}\left(x_{n}-x_{i}\right)\end{array}\right]\left[\begin{array}{c}c_{o} \\ c_{1} \\ c_{2} \\ c_{3} \\ . . \\ c_{n}\end{array}\right]$

Taken into account that the last data points $\left(x_{n}, y_{n}\right)$ is used for determination of the last coefficient $c_{n}$, as identified below. For this $n^{\text {th }}$ degree polynomial $N_{n}(x)$ to pass all $n+1$ points ( $x_{i}$, $\left.y_{i}\right),(i=0, \ldots n)$ it needs to satisfy the following $n+1$ equations, which can also be described in matrix form. The $n+1$ coefficients $c_{o}, \ldots . . c_{n}$ could be estimated by solving these $n+1$ equations in the triangular equations system progressively from top to down (Qiao et al., 2018).

$$
\begin{aligned}
& c_{o}=y_{o}=f\left(x_{o}\right)=f\left[x_{o}\right] \\
& c_{1}=\frac{y_{1}-y_{o}}{x_{1}-x_{o}}=f\left[x_{o}, x_{1}\right]
\end{aligned}
$$




$$
\begin{aligned}
& c_{2}=\frac{\frac{y_{2}-y_{o}}{x_{2}-x_{o}}-\frac{y_{1}-y_{o}}{x_{1}-x_{o}}}{x_{2}-x_{1}} \\
& =f\left[x_{o}, x_{1}, x_{2}\right]
\end{aligned}
$$

$$
\left[\begin{array}{cccccc}
x_{o}^{n} & x_{o}^{n-1} & x_{o}^{n-2} & \cdots & x_{o} & 1 \\
x_{1}^{n} & x_{1}^{n-1} & x_{1}^{n-2} & \cdots & x_{1} & 1 \\
x_{2}^{n} & x_{2}^{n-1} & x_{2}^{n-2} & \cdots & x_{2} & 1 \\
\vdots & \vdots & \vdots & \vdots & \vdots & \vdots \\
x_{n}^{n} & x_{n}^{n-1} & x_{n}^{n-2} & \cdots & x_{n} & 1
\end{array}\right]\left[\begin{array}{c}
a_{n} \\
a_{n-1} \\
a_{n-2} \\
a_{n-3} \\
\vdots \\
a_{0}
\end{array}\right]=\left[\begin{array}{c}
y_{o} \\
y_{1} \\
y_{2} \\
\vdots \\
y_{n}
\end{array}\right]
$$

In general, we have [7]:

$$
c n=f[x o, \ldots \ldots x n]=\sum_{j=0}^{n} \frac{f\left(x_{j}\right)}{\amalg_{i=0, i \neq j}^{n}\left(x_{j}-x_{i}\right)}
$$

\section{$B$. The Lagrange Interpolation}

In statistical and numerical analysis, Lagrange methods are used for polynomial interpolation. For a given number of points $\left(x_{j}, y_{j}\right)$, the Lagrange polynomial is the polynomial of lowest degree which supposes the value $y_{j}$ at each value $x_{j}$, and it can be calculated using equations from (11) to (17) (Sun et al., 2015).

$$
\begin{gathered}
\hat{y}(x)=\sum_{i=1}^{N} y_{i} f_{i}(x) \\
{\left[\begin{array}{c}
f_{1}(x) \\
\ddot{\prime} \\
f_{N}(x)
\end{array}\right]=\left[\begin{array}{ccc}
F_{1}\left(x_{1}\right) & \cdots & F_{1}\left(x_{N}\right) \\
\vdots & \ddots & \vdots \\
F_{N}\left(x_{1}\right) & \cdots & F_{N}\left(x_{N}\right)
\end{array}\right]^{-1}\left[\begin{array}{c}
F_{1}(x) \\
\vdots \\
F_{N}(x)
\end{array}\right]} \\
y=a_{o}+a_{1} x+a_{2} x^{2}+a_{3} x^{3}
\end{gathered}
$$

Which can be generalized in Lagrange form as below (Sun $e t$ al., 2015):

$$
\begin{gathered}
L_{i}(x)=\prod_{\substack{k=1 \\
k \neq 1}}^{n} \frac{x-x_{k}}{x_{i}-x_{k}} \\
p(x)=\sum_{j=0}^{n} y_{i} L_{n, j}(x)
\end{gathered}
$$

\section{Least Square Interpolation}

Nowadays, LSM has been most widely used in data fitting. The commonly used basis functions are polynomials (White et al., 2007). The local approximants of the LSM only take the random errors of the dependent variable into account,
This matrix can be expressed in equation form as follow:

$$
\begin{aligned}
y(x)= & y_{o} \frac{\left(x-x_{1}\right)\left(x-x_{2}\right)\left(x-x_{3}\right)}{\left(x_{o}-x_{1}\right)\left(x_{o}-x_{2}\right)\left(x_{o}-x_{3}\right)} \\
+ & y_{1} \frac{\left(x-x_{o}\right)\left(x-x_{2}\right)\left(x-x_{3}\right)}{\left(x_{1}-x_{o}\right)\left(x_{1}-x_{2}\right)\left(x_{1}-x_{3}\right)} \\
& +y_{2} \frac{\left(x-x_{o}\right)\left(x-x_{1}\right)\left(x-x_{3}\right)}{\left(x_{2}-x_{o}\right)\left(x_{2}-x_{1}\right)\left(x_{2}-x_{3}\right)} \\
& +y_{3} \frac{\left(x-x_{o}\right)\left(x-x_{1}\right)\left(x-x_{2}\right)}{\left(x_{3}-x_{o}\right)\left(x_{3}-x_{1}\right)\left(x_{3}-x_{2}\right)}
\end{aligned}
$$

whereas the independent variables of measurement data always contain errors (Barchiesi \& Grosges, 2017). It is one of the most popular approach used to identify the trend line of the results. In this methodology, a mathematical relation is built between the obtained variables and the time factors.

Let $\left(t_{1}, y_{1}\right),\left(t_{2}, y_{2}\right), \ldots,\left(t_{n}, y_{n}\right)$ indicate the resultant time series. In this methodology the trend values $y_{c}$ of the variable $y$ are calculated so as to satisfy the sum of the variations of $y$ from their identical trend values is zero, and also to satisfy the sum of the squares of the variations of $y$ from their identical trend values (Grabe, 2001).

LSM might be categorized into two types: nonlinear least squares, and ordinary least squares, it is depending on the linearity of the residuals. For linear LSM equations the solution is a closed-form. The nonlinear equations are always resolved by iterations elaboration.

LSM might also be approved as a method of moments estimator (Gouda \& Riham, 2017).

To fit the nonlinear function: $y=a+b x+c x^{2}$ :

Solve the following equations for $a, b, c$ :

$$
\begin{gathered}
\sum y=n a+b \sum x+c \sum x^{2} \times \sum x y=a \sum x \\
+b \sum x^{2}+c \sum x^{3}
\end{gathered}
$$

\section{METHOD VERIFICATION}

In this article method verification is required for the three methods used (LSM, LA, NA). The aim of this verification is to evaluate the accuracy of each one. To verify the result of those methods the deviation should be calculated. This deviation can be expressed as the difference between the 
experimental results and the calculated results from the produced polynomial equations as expressed in Equation (20) (Kühne et al., 2014):

$$
e(x)=y(x)-\hat{y}(x)
$$

In this paper the authors have investigated the difference between least square, Lagrange, Newton forward methods for generating the interpolation equations. Two cases studies were used to prove the conclusion of this article, reference force proving instruments and reference pressure sensor. The error for each method has been calculated to identify which statistical method is the most accurate and reliable.

\section{EXPERIMENTAL SETUP}

The first case study is $10 \mathrm{kN}$ force transducer (series $\mathrm{K} \mathrm{T} \mathrm{N} \mathrm{-}$ D, GTM manufactured) and it was calibrated by $50 \mathrm{kN}$ Dead weights standard machine (GTM Manufactured). This machine is internationally recognized by BIPM with uncertainty of $\pm 0.002 \%$. The $10 \mathrm{kN}$ force transducer is calibrated in accordance with ISO 376:2011, [ISO 376:2011], and classified as Class 0 with uncertainty of $\pm 0.02 \%$. The experiment setup is shown in Figure 1. The results of $10 \mathrm{kN}$ force transducer were monitored by DMP40-digitalprecision-measuring-amplifier (HBM manufactured) with resolution of $0.000001 \mathrm{mv} / \mathrm{v}$ to get the best performance and the highest accuracy and better uncertainty, refer to Figure 2.

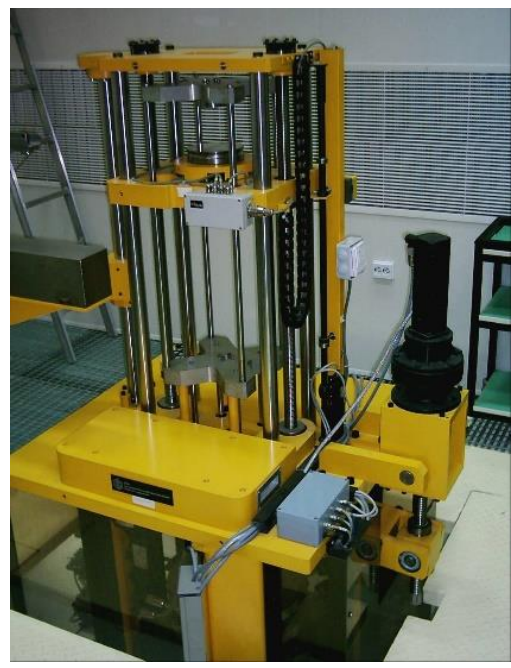

Figure 1. Standard dead weights machine used (5o kN)

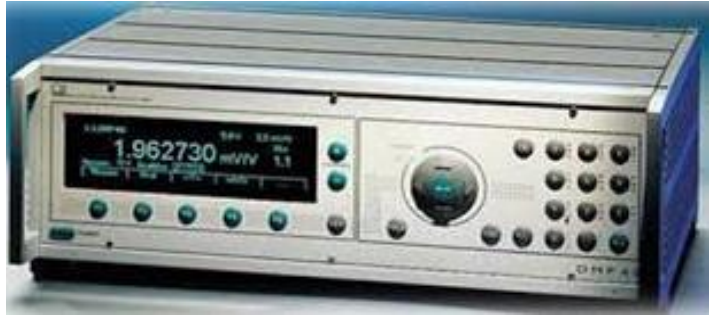

Figure 2. DMP40 amplifier used in the experimental work

The second case study is a pressure transmitter (HBM manufactured) with relative resistance change output $(2 \mathrm{mV} / \mathrm{V})$. This pressure transmitter was calibrated using standard pressure dead weight tester (Desgranges \& Huot manufactured) in accordance with Guidelines on the Calibration of Electromechanical and Mechanical manometers and EURAMET Calibration Guide No. 17 Version 3.o. The calibration of this pressure transmitter is traceable to NIS which realize SI units through international recognition. The results of pressure transmitter were monitored by DMP40, Figure 3 shows the experimental setup of the pressure transmitter with the pressure dead weight tester.

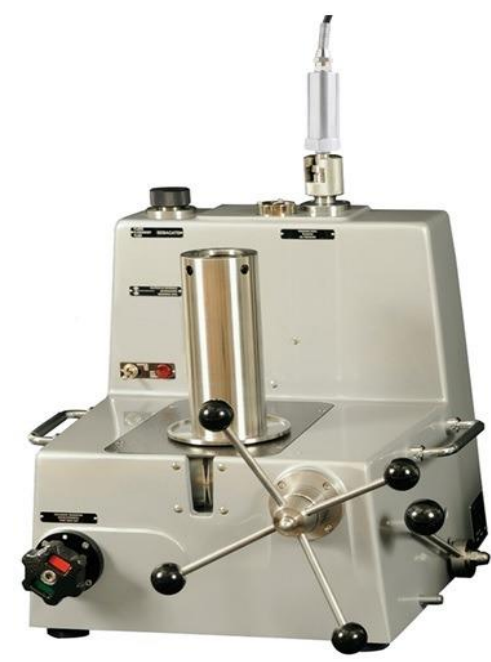

Figure 3. Pressure transmitter setup

\section{RESULTS AND DISCUSSION}

\section{A. Force Proving Instruments}

The polynomial equation is obtained from statistical analysis of the calibration results. Four series of readings at 10-points loads from 1 to $10 \mathrm{kN}$, The response of the force proving instrument is recorded and the produced equation was 
generated. The general equation for force proving instruments is stated as follow:

$$
R=x \times F+y \times F^{2}+z \times F^{3}
$$

where

$$
\begin{aligned}
& R: \text { is the calculated response }(\mathrm{mV} / \mathrm{V}) \\
& F: \text { is the applied force }(\mathrm{kN})
\end{aligned}
$$

$x, y, z$ are constants and their values as below:

\begin{tabular}{|c|c|c|c|}
\hline Method & $\boldsymbol{x}$ & $\boldsymbol{y}$ & $\boldsymbol{z}$ \\
\hline LSM & $2.0008 \mathrm{E}-01$ & $-1.18238 \mathrm{E}-05$ & $5.73109 \mathrm{E}-\mathrm{O} 7$ \\
\hline LA & $2.0006 \mathrm{E}-01$ & $-6.13500 \mathrm{E}-05$ & $1.76167 \mathrm{E}-05$ \\
\hline NA & $2.00121 \mathrm{E}-01$ & $-2.15500 \mathrm{E}-04$ & $3.62500 \mathrm{E}-05$ \\
\hline
\end{tabular}

The three methods results can be summarized in Table 1 .

Table 1. The three methods of interpolation equation produced for force transducer

\begin{tabular}{cccccccc}
\hline \multirow{2}{*}{$\begin{array}{c}\text { Applied } \\
\text { Force }\end{array}$} & $\begin{array}{c}\text { Measured } \\
\text { average }\end{array}$ & \multicolumn{2}{c}{ Calculated response** } & \multicolumn{3}{c}{ Absolute error } \\
\cline { 3 - 8 } response & LA & LSM & NA & LA & LSM & NA \\
\hline $\mathrm{kN}$ & $\mathrm{mV} / \mathrm{V}$ & $\mathrm{mV} / \mathrm{V}$ & $\mathrm{mV} / \mathrm{V}$ & $\mathrm{mV} / \mathrm{V}$ & $\mathrm{mV} / \mathrm{V}$ & $\mathrm{mV} / \mathrm{V}$ & $\mathrm{mV} / \mathrm{V}$ \\
1 & 0.200018 & 0.200139 & 0.200067 & 0.199942 & 0.000121 & 0.000049 & 0.000077 \\
2 & 0.400040 & 0.400506 & 0.400114 & 0.399669 & 0.000466 & 0.000074 & 0.000371 \\
3 & 0.600129 & 0.601207 & 0.600145 & 0.599402 & 0.001078 & 0.000016 & 0.000727 \\
4 & 0.800212 & 0.802348 & 0.800161 & 0.799355 & 0.002136 & 0.000051 & 0.000857 \\
5 & 1.000263 & 1.004035 & 1.000168 & 0.999748 & 0.003772 & 0.000094 & 0.000515 \\
6 & 1.200122 & 1.206372 & 1.200169 & 1.200797 & 0.006250 & 0.000047 & 0.000674 \\
7 & 1.400236 & 1.409467 & 1.400166 & 1.402719 & 0.009231 & 0.0000697 & 0.002484 \\
8 & 1.600083 & 1.613424 & 1.600164 & 1.605734 & 0.013341 & 0.000081 & 0.005651 \\
9 & 1.800092 & 1.818349 & 1.800166 & 1.810058 & 0.018257 & 0.000074 & 0.009965 \\
10 & 2.000243 & 2.024349 & 2.000175 & 2.015908 & 0.024106 & 0.000068 & 0.015665 \\
\hline
\end{tabular}

** Calculated from Equation (21) 


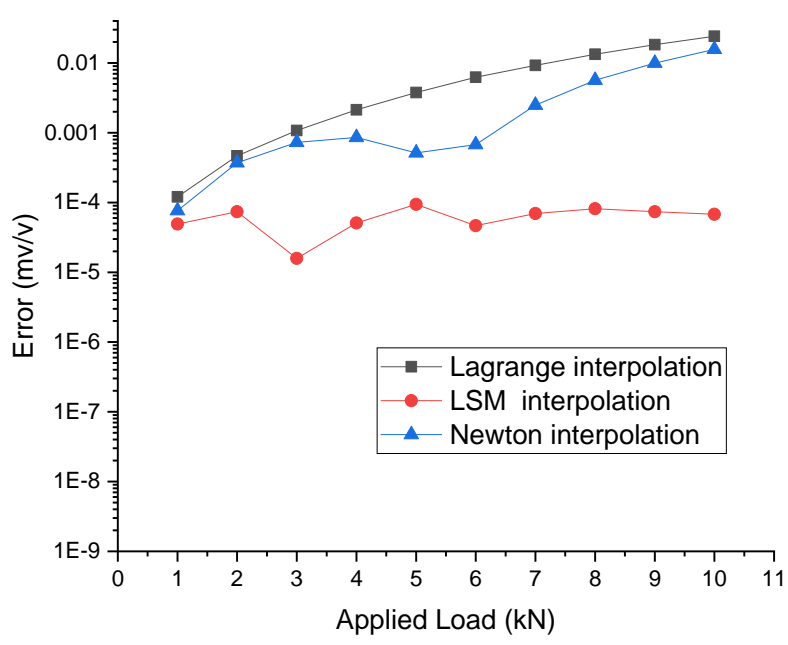

\begin{tabular}{|l|c|c|c|}
\cline { 2 - 4 } \multicolumn{1}{c|}{} & $\boldsymbol{x}$ & $\boldsymbol{y}$ & $\boldsymbol{z}$ \\
\hline LSM & $4.003375 \mathrm{E}-03$ & $-2.484189 \mathrm{E}-09$ & $3.503110 \mathrm{E}-12$ \\
\hline LA & $4.005085 \mathrm{E}-03$ & $-2.909524 \mathrm{E}-08$ & $1.033045 \mathrm{E}-10$ \\
\hline NA & $4.005084 \mathrm{E}-03$ & $-3.938422 \mathrm{E}-08$ & $1.031242 \mathrm{E}-10$ \\
\hline
\end{tabular}

$$
R=x \times P+y \times P^{2}+z \times P^{3}
$$

\section{where}

$\mathrm{R}$ : is the calculated response $(\mathrm{mv} / \mathrm{v})$

$\mathrm{P}:$ is the applied pressure (bar)

$\mathrm{x}, \mathrm{y}, \mathrm{z}$ are constants and their values as below

Figure 4. Representing the error for the different methods used in this investigation for force transducer

\section{B. For Pressure Reference Transmitter}

The polynomial equation is obtained from statistical analysis of the calibration results. three series of readings at 10-points loads from 50 to 500 bar. The response of the pressure proving instrument is recorded and the produced equation was generated. The general equation for pressure proving instrument is stated as follow

Table 2. The three methods of interpolation equation produced for pressure transducer

\begin{tabular}{cccccccc}
\hline & \multirow{2}{*}{$\begin{array}{c}\text { Average } \\
\text { Applied }\end{array}$} & \multicolumn{2}{c}{ Calculated response** } & & \multicolumn{2}{c}{ Absolute error } \\
\cline { 3 - 9 } Pressure & response & LA & LSM & NA & LA & LSM & NA \\
\hline bar & $\mathrm{mv} / \mathrm{v}$ & $\mathrm{mv} / \mathrm{v}$ & $\mathrm{mv} / \mathrm{v}$ & $\mathrm{mv} / \mathrm{v}$ & $\mathrm{mv} / \mathrm{v}$ & $\mathrm{mv} / \mathrm{v}$ & $\mathrm{mv} / \mathrm{v}$ \\
50.0129 & 0.200246 & $0.20039 \mathrm{v} 2$ & 0.200215 & 0.200220 & 0.000146 & 0.0000315 & 0.0000258 \\
100.044 & 0.400497 & 0.401079 & 0.400492 & 0.400394 & 0.000582 & 0.0000047 & 0.0001033 \\
150.0749 & 0.600757 & 0.602067 & 0.600762 & 0.600524 & 0.001311 & 0.0000055 & 0.0002324 \\
200.1054 & 0.801015 & 0.803432 & 0.801026 & 0.800688 & 0.002417 & 0.0000107 & 0.0003266 \\
250.1352 & 1.001279 & 1.0052500 & 1.001284 & 1.000962 & 0.003971 & 0.0000048 & 0.0003173 \\
300.1645 & 1.201533 & 1.2075995 & 1.201542 & 1.201424 & 0.006067 & 0.0000089 & 0.0001085 \\
350.1934 & 1.401804 & 1.410559 & 1.401801 & 1.402153 & 0.008755 & 0.0000025 & 0.0003492 \\
400.2220 & 1.602078 & 1.614206 & 1.602065 & 1.603226 & 0.012129 & 0.0000123 & 0.0011476 \\
450.248 & 1.802340 & 1.818611 & 1.802329 & 1.804711 & 0.016271 & 0.0000108 & 0.0023713 \\
500.2750 & 2.002594 & 2.023860 & 2.002605 & 2.006699 & 0.021267 & 0.0000118 & 0.0041049 \\
\hline
\end{tabular}

** Calculated from Equation (22) 


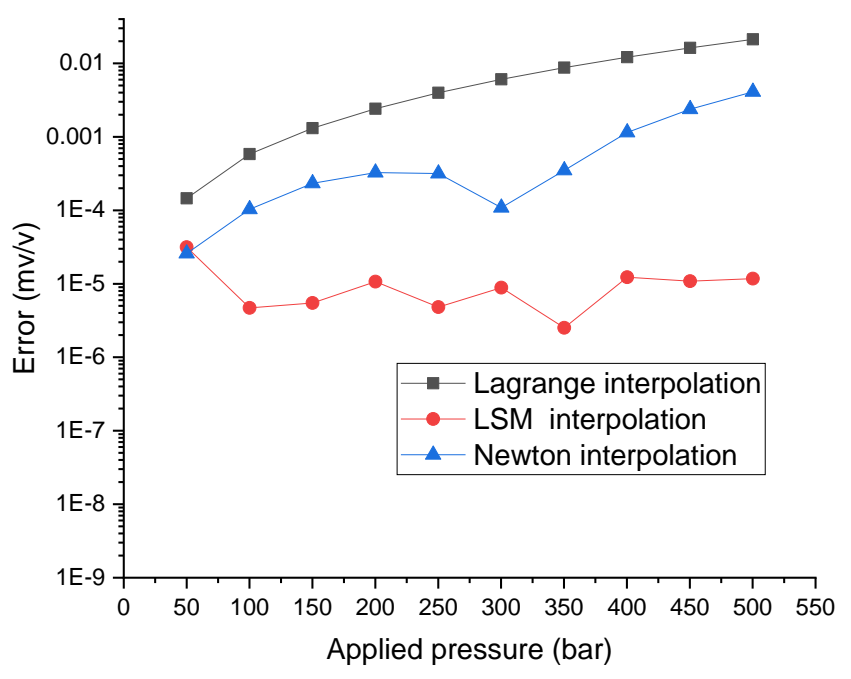

Figure 5. Represents the error for the different methods used in this investigation for pressure transducer

In the previous Tables 1 and 2, and Figures 4 and 5 the results of this investigation were monitored. From this investigation, the errors in response comparing with LSM, LA, NA were calculated. This error was calculated as the difference between the actual measured response and the calculated response. It was observed that all of the above methods are used to generate the interpolation equation in various manners. From the previous tables and figures, it was observed that LSM is the more realistic and more accurate approach to find the interpolation equation. For the Newton method it is a simple formula for the remnant of the interpolation and it easier to be used to find intermediate point without identification of the coefficients all over again. Regarding the accuracy of each method it was noted, that LSM is the most accurate approach to generate the polynomial function.

\section{CONCLUSION}

This article is not only a comparison between statistical interpolation methods, but it is rooted to the most accurate and realistic statistical method for generating and the interpolation polynomial equations for the measuring devices. Three methods were used to find the calibration equation for different measuring devices. The results of this investigation were verified based on actual results of reference sensors (force and pressure transducer). The error for each method was calculated comparing with the actual response produced from actual calibration. It was concluded that the Least square method is the most accurate and the most realistic method to find the interpolation equation, in comparison with Newton and Lagrange method which is the least accurate. For future it is recommended to investigate the effects of those approached on the uncertainty budget especially the interpolation error produced.

\section{REFERENCES}

Barchiesi, D \& Grosges, T 2017, 'Propagation of uncertainties and applications in numerical modeling: tutorial', Journal of the Optical Society of America, vol. 34, no. 9, pp. 1602-1619.

Grabe, $M$ 2001, 'Estimation of measurement uncertainties-an alternative to the ISO guide', Metrologia, vol. 38, no. 2, pp. 97.

Gu, T, Tu, Y, Tang, D, Lin, S \& Fang, B 2020, 'A trimmed moving total least-squares method for curve and surface fitting', Measurement Science and Technology, vol. 31, no. 4, pp. 045003.

Han, CT \& Gaik, TK 2016, 'Newton's divided difference interpolation using scientific calculator' in AIP Conference Proceedings, vol. 1775, no. 1, pp. 030102, October 2016, AIP Publishing LLC.
ISO 376:2011 Metallic materials - Calibration of forceproving instruments used for the verification of uniaxial testing machines.

Kühne, M, Hilbrunner, F, Büchner, H, Jäger, G, Manske, E, Fröhlich, T 2014, 'Traceable measurement of mechanical parameters of double bending beam force transducers according to EN ISO 376', Measurement, vol. 51, pp. 336-342.

Li, BZ, Zhang, YL, Wang, X \& Cheng, QY 2016, 'A new method for Chebyshev polynomial interpolation based on cosine transforms', Circuits, Systems, and Signal Processing, vol. 35, no. 2, pp. 719-729.

Mahmoud, GM \& Hegazy, RS 2017, 'Comparison of GUM and Monte Carlo methods for the uncertainty estimation in hardness measurements', International 
Journal of Metrology and Quality Engineering, vol. 8, pp. 14 .

Qiao, W, Wei, Z, Yonggang, C, Gang, M, Xiaolin, C, Yu, M, Chen, E 2018, 'Regularized moving least-square method and regularized improved interpolating moving least-square method with nonsingular moment matrices', Applied Mathematics and Computation, vol. 325, pp. 120-145.

Rainer K 1998, "Numerical Analysis" Part of the graduate texts in mathematics book series (GTM, volume 181), Springer, New York, US.

Ramesh KM 2012, 'Note on Newton interpolation formula', International Journal of Math. Analysis, vol. 6, no. 50, pp. 2459-2465.

Srivastava, RB \& Purushottam, KS 2012, 'Comparison of Lagrange's and Newton's interpolating polynomials', Journal of Experimental Sciences, vol. 3, no. 1.

Sun, FX, Wang, JF, Cheng, YM \& Huang, AX 2015, 'Error estimates for the interpolating moving leastsquares method in n-dimensional space', Applied Numerical Mathematics, vol. 98, pp. 79-105.

White, DR \& Saunders, P 2007, 'The propagation of uncertainty with calibration equations', Measurement Science and Technology', vol. 18, pp. 2157-2169. 